\title{
SAMPLE EXAMINATION QUESTIONS
}

1. Explain briefly what is meant by each of the following:

(i) Euler path

(ii) fixed point theorem

(iii) four color problem

(iv) unit braid of order $n$

(v) Móbius band.

2. Give an example of each of the following:

(i) a map which cannot be colored in three colors

(ii) a map which has no Hamilton path

(iii) a closed curve which is not simple

(iv) a transformation which has no fixed point

(v) a surface having Euler characteristic zero.

3. Complete the following table:

\begin{tabular}{|l|l|l|l|l|l|}
\hline & V & E & F & n & $r$ \\
\hline regular tetrahedron & 4 & & & & \\
\hline cube & & 12 & & & \\
\hline octahedron & & & 8 & & \\
\hline dodecahedron & & & & 5 & \\
\hline icosahedron & & & & & 5 \\
\hline
\end{tabular}

4. (i) Convert 39 and 56 to base 2 and multiply the se numbers, either by multiplication in base 2 or by the so called Russian method.

(ii) Use mathematical induction to prove that

$$
2^{1}+2^{2}+\ldots+2^{n}=2^{n+1}-2 \text {. }
$$

5. Evaluate each of the following:
(i) $\pi(15)$,
(ii) $\sigma(15)$,
(iii) $\phi(15)$
(iv) $(15,25)$
(v) ( 3$)$. 
6. Explain what is meant by each of the following and describe the present state of these problems:
(i) Goldbach's problem
(ii) Waring's problem
(iii) Fermat's

last theorem (iv) Euler's problème de rencontres (v) the stamp folding problem.

7. Define each of the following:

(i) prime pair (ii) perfect number (iii) amicable numbers

(iv) primitive Pythagorean triplet (v) googolplex.

8. (i) What is meant by the statement that two sets have the same potency?

(ii) Prove that the set of integers and the set of rationals have the same potency.

(iii) What is the continuum hypothesis?

9. (i) Find the equation of the line through $(1,2)$ having slope 3 .

(ii) Find the equation of the circle of radius 3 having center at $(1,2)$.

(iii) What is the distance between $(1,2,3)$ and $(3,2,1)$ ?

(iv) Give coordinates of an equilateral triangle of side 1.

10. Show how two unit squares may be dissected and the pieces reassembled to form a single square.

Can the problem above be solved if "two" is replaced by "five"?

11. (i) Discuss various approaches to $\mathrm{n}$ dimensional geometry.

(ii) Give the number of vertices, edges, faces, etc. in a five dimensional cube. 
12. (i) What is the largest area which can be enclosed by a fence $100 \mathrm{ft}$. long?

(ii) Two parallel roads are 1 mile apart. Two houses 1 mile apart are situated half-way between the roads. Find the length of a shortest path leading from one house to the other and touching both roads.

13. (i) Find the point the sum of whose distances from $(0,0)$, $(2,0),(2,4)$ and $(1,1)$ is as small as possible.

(ii) Calculate this minimal distance.

14. Describe geodesics on each of the following surfaces:
(i) plane
(ii) sphere
(iii) cylinder (iv) cube.

15. Describe extremal properties of each of the following:

(i) cube (ii) sphere (iii) great circle (iv) cycloid.

16. Give addition and multiplication tables for the integers modulo 5. State the basic rules of algebra which are satisfied by this system. Is it true in this system that for all $a, b, c, a b=a c$ implies $b=c$ ?

17. Define each of the following:

(i) ellipse (ii) general cone (iii) golden section (iv) plane of symmetry (v) trapezoid (vi) cycloid (vii) regular tetrahedron (viii) velocity (ix) acceleration (x) hyper-plane.

18. Comment (about one page) on one of the following statements:

(i) Mathematics may be defined as the subject in which we never know what we are talking about, nor whether what we àre saying is true.

(ii) There is probably no other science which presents such different appearances to one who cultivates it and one who does not, as mathematics. 
19. Discuss briefly some questions on which there is a significant difference of opinion among competent mathematicians.

20. Name mathematicians associated with each of the following:

(i) intuitionism (ii) proof of the prime number theorem

(iii) solution of Waring's problem (iv) applications of mathematics to economics (v) cybernetics.

21. State at least one contribution to mathematics of each of the following:

(i) Eratosthenes (ii) Fermat (iii) Leibnitz (iv) Gauss

(v) Galois (vi) Riemann (vii) Russell (viii) Gödel (ix)

Vinogradov $(x)$ Courant.

22. (i) Determine the triangle of largest area which will fit inside a semi-circle of radius 1 .

(ii) For what values of $x$ does $2 x^{3}-9 x^{2}+2$ assume a relative extreme value?

(iii) Find the area bounded by $y=2 x^{2}$, the $x$ - axis, and the line $x=1$.

(iv) Use calculus to find the volume of a sphere of radius 1 .

23. What is meant by each of the following formulae:

(i) $\frac{d}{d x}(f(x)+g(x))=\frac{d f(x)}{d x}+\frac{d g(x)}{d x}$.

(ii) $\mathrm{v}=\frac{\mathrm{d} s}{\mathrm{dt}}$.

(iii) $\frac{d}{d x} \int f(x) d x=f(x)$.

(iv) $A_{x}=\pi \int_{a}^{b} y^{2} d x$.

(v) $W=\int_{a}^{b} F(s) d s$. 
24. Write a short note on the relation between mathematics and each of the following:

(i) physics (ii) philosophy (iii) ethics (iv) numerology.

25. Which of the following statements are true and which are false?

(i) Pure mathematics has suffered a decline in recent years.

(ii) Exceptional ability in mathematics implies exceptional ability in computation.

(iii) Spinoza used the postulational approach in his study of ethics.

(iv) Checker problems require mathematical reasoning for their solution.

(v) Modern mathematics has important applications to bookke eping.

(vi) Mathematical induction can be used to prove that the sun will rise tomorrow.

(vii) A statement concerning all positive integers can sometimes be disproved by considering the first $1,000,000$ cases.

(viii) Topology has been applied to some problems of psychology.

(ix) To be a successful engineer one must be familiar with at least $25 \%$ of modern mathematics.

(x) This statement is false.

University of Alberta 